山्ل FRANÇAISE

$\gg \mathrm{DE}$

필 PÉDAGOGIE

\section{Revue française de pédagogie}

Recherches en éducation

192 | juillet-août-septembre 2015

Face aux mutations des marchés de l'emploi, quelles

politiques de formation?

\title{
Perception des pratiques pédagogiques des enseignants par les étudiants de première année universitaire et effets sur leur scolarité
}

Perception of lecturers' pedagogical practices by first year university students and their effect on their studies

\section{Amélie Duguet}

\section{OpenEdition}

Journals

Édition électronique

URL : http://journals.openedition.org/rfp/4839

DOI : $10.4000 / \mathrm{rfp} .4839$

ISSN : 2105-2913

\section{Éditeur}

ENS Éditions

\section{Édition imprimée}

Date de publication : 30 septembre 2015

Pagination : 73-94

ISSN : 0556-7807

\section{Référence électronique}

Amélie Duguet, « Perception des pratiques pédagogiques des enseignants par les étudiants de première année universitaire et effets sur leur scolarité », Revue française de pédagogie [En ligne], 192 | juillet-août-septembre 2015, mis en ligne le 30 septembre 2018, consulté le 03 janvier 2020. URL : http://journals.openedition.org/rfp/4839; DOI : 10.4000/rfp.4839 


\title{
Perception des pratiques pédagogiques des enseignants par les étudiants de première année universitaire et effets sur leur scolarité
}

\author{
Amélie Duguet
}

\begin{abstract}
En France, peu de recherches se sont penchées sur les effets du contexte universitaire et plus particulièrement des pratiques pédagogiques telles que perçues par les étudiants entrant en première année universitaire sur leur scolarité. Aussi ce travail se propose-t-il d'étudier l'effet de la perception de ces pratiques par les étudiants sur leur motivation, leurs manières d'étudier et leur réussite. À l'appui de données recueillies par questionnaires auprès de 734 étudiants inscrits en première année universitaire au sein de cinq filières, il est montré que la perception des pratiques par les étudiants influe particulièrement sur leur motivation et par ce biais, de façon indirecte, sur leurs manières d'étudier et leur réussite.
\end{abstract}

Mots-clés (TESE) : pratique pédagogique, motivation, université, étudiant du premier cycle des études supérieures.

\section{Introduction}

En France, le système d'enseignement supérieur présente la particularité d'être constitué d'un grand nombre de structures et de diplômes. Parmi ceux-ci, l'université représente à l'heure actuelle l'institution qui accueille le plus grand nombre de jeunes bacheliers. En raison notamment de la démocratisation et de la massification de l'accès aux études universitaires au cours $\mathrm{du} \mathrm{xx}^{\mathrm{e}}$ siècle, elle rassemble aujourd'hui à elle seule plus de la moitié des individus inscrits dans le supérieur. L'arrivée de ce public de plus en plus hétérogène, à la fois socialement et scolairement, a contribué à faire évoluer les finalités de l'enseignement supérieur. L'objectif n'est plus uniquement de former une «élite scolaire», mais de participer à la réduction 
des inégalités sociales (Michaut, 2000), I'université étant la seule institution d'enseignement supérieur interdite de procéder à une sélection à l'entrée (Convert, 2010). Toutefois, cette situation a également eu pour conséquence une hausse croissante des taux d'échec. Il est à dire que l'université constitue une orientation par défaut pour bon nombre d'individus n'ayant pas été acceptés au sein des filières sélectives (Ryk, 2012), une «roue de secours» (Beaud, 2008) pour ceux qui n'ont encore aucun projet professionnel précis et parfois même une alternative pour ceux qui ne souhaitent pas entrer immédiatement dans la vie active (Beaud \& Pialoux, 2001). Par conséquent, parmi les individus entrés en première année à la rentrée 2011, seuls $43,8 \%$ sont passés en deuxième année tandis que $29 \%$ ont redoublé et $27,2 \%$ ont quitté le système universitaire (MESR, 2013). Certes, comme l'évoque Millet (2012), ces taux sont à relativiser. Cette situation a néanmoins conduit les chercheurs depuis maintenant plusieurs décennies à tenter d'expliquer ce phénomène en examinant le poids exercé par un certain nombre de facteurs sur la réussite. Ces travaux mettent en évidence l'importance du passé scolaire de l'étudiant sur sa réussite. Ainsi, les bacheliers généraux présentent de meilleurs taux de réussite à l'université que les bacheliers technologiques, eux-mêmes plus performants que les bacheliers professionnels (Michaut, 2000; Morlaix \& Suchaut, 2012). Parmi les bacheliers généraux, ceux qui sont détenteurs d'un baccalauréat scientifique bénéficient d'une "voie royale» pour continuer les études dans l'enseignement supérieur (Beaupère, Chalumeau, Gury et al., 2007). L'obtention d'une mention très bien au baccalauréat plutôt que passable multiplie par trois la probabilité de conclure une année de premier cycle (Gruel, 2002). En revanche, un redoublement dans le secondaire triple les risques de sortir sans diplôme de l'enseignement supérieur (Gury, 2007). Outre ces variables scolaires, le fait d'exercer une activité rémunérée en dehors des heures de cours peut s'avérer préjudiciable pour la réussite, particulièrement si cette activité entre en concurrence avec les études (Béduwé \& Giret, 2004).

La motivation de l'étudiant a elle aussi toute son importance puisqu'elle joue "un rôle capital dans les activités d'apprentissage, de créativité, de performance et de persévérance dans les études » (Vallerand, 1993). L'effet de cette variable sur la réussite diffère en fonction du degré de motivation de l'individu : tandis que la motivation intrinsèque joue un rôle positif et significatif sur la moyenne annuelle, l'amotivation, ou autrement dit l'absence de motivation, exerce un impact négatif sur les résultats obtenus par les étudiants aux examens. Cet effet varie également en fonction du niveau scolaire des étudiants : il tend à être moins important pour les individus les plus faibles (Lambert-Le Mener, 2012). Des recherches ont également attesté le «rôle prépondérant des modalités d'études comme facteur de discrimination et de réussite» (Alava, 2000). De façon globale, les chercheurs (Frickey \& Primon, 2003 ; Soulié, 2002) s'accordent à dire que bien qu'étant amenées à varier d'une matrice disciplinaire à l'autre (Millet, 2003), les manières d'étudier favorisant un travail en profondeur de la matière sont davantage bénéfiques à la réussite que celles visant un travail de surface.

Si la prise en compte de ces facteurs inhérents aux étudiants est nécessaire pour une meilleure compréhension de la réussite, l'apprentissage ne relève toutefois pas uniquement de l'apprenant (Delhaxhe, Houart \& Pollet, 2011) et il est important de ne pas négliger «les causes d'échec inhérentes au fonctionnement même de l'université » (Duru-Bellat, 1995). C'est ainsi qu'ont été produits des travaux sur les effets de site (Felouzis, 2001), sur les inégalités territoriales (Nicourd, Samuel \& Vilter, 2011), sur les dispositifs d'aide aux étudiants (M'Piayi, 1999), sur les effets du tutorat d'accompagnement (Danner, 1999), ou bien encore sur l'efficacité des modes d'organisation pédagogique (Michaut, 2000). Néanmoins, peu de recherches ont porté, en France, sur le rôle des enseignants et plus précisément de leurs pratiques pédagogiques sur la scolarité des étudiants. Tout se passe en réalité comme si la littérature scientifique supposait de façon implicite que l'échec tient davantage aux caractéristiques des étudiants qu'à celles du contexte d'étude (Galand, Neuville \& Frenay, 2005). Pourtant, certains évoquent la nécessité, pour lutter contre l'échec, de faire évoluer la pédagogie mise en œuvre à l'université (Romainville, 2000), puisqu'elle constituerait un «facteur déterminant pour la réussite des étudiants» (Bertrand, 2014). Se pose de fait ici la question de la qualité pédagogique des formations proposées par les universités et de l'efficacité des modes d'enseignement. Or, si la recherche en pédagogie universitaire est en France actuellement en pleine expansion, elle reste «peu structurée, peu lisible, peu visible» (Bertrand, 2014). Peu de travaux ont de surcroît été consacrés au sein de I'hexagone aux pratiques pédagogiques des enseignants universitaires ainsi qu'à leurs effets sur les étudiants (De Ketele, 2010). C'est par conséquent dans 
cette perspective que se positionne cet article, dont l'objectif est d'apporter un nouvel éclairage sur la manière dont ces pratiques peuvent, à travers la manière dont elles sont perçues par les étudiants, influer sur leur scolarité.

\section{Les pratiques pédagogiques : quel(s) type(s) d'influence sur la scolarité des étudiants?}

Par «pratique pédagogique», entendons l'ensemble des actions mises en œuvre par l'enseignant durant les cours, de manière plus ou moins consciente, en vue de faire acquérir des connaissances aux étudiants. Or, ces pratiques peuvent avoir un impact négatif sur l'apprentissage si l'enseignant n'a pas d'«habiletés pédagogiques» et s'il n'est pas «concret» (Ménard, 2012). Elles constitueraient même un facteur de «rétention » (Fontaine \& Peters, 2012). D'autres travaux, certes anciens, indiquent que les pédagogies dites actives seraient plus propices au développement d'une motivation intrinsèque chez les étudiants (Sénécal, Vallerand \& Pelletier, 1992, cités par Forner \& Simonot, 2001) et donc plus motivantes (Harter, 1981). A contrario, la pédagogie «traditionnelle» et en ce sens les pratiques qualifiées de «contrôlantes » généralement employées par les enseignants durant les cours magistraux conduisent au développement d'une motivation extrinsèque (Solomon \& Kendall, 1976). De plus, des chercheurs tels que Deci et Ryan (1985), Bandura (1997) ou encore Viau (1998)', en adoptant une approche sociocognitive du concept de motivation, laissent supposer que l'environnement d'apprentissage et par là même les pratiques des enseignants exercent un effet sur les besoins, croyances et perceptions des étudiants se situant à l'origine de leur motivation. Les pratiques des enseignants auraient également des conséquences

1 Si elles ne portent pas explicitement sur l'enseignement supérieur, les théories de ces auteurs ont néanmoins largement été reprises par les chercheurs traitant de la motivation des étudiants dans l'enseignement universitaire, à l'image d'une part des travaux de Forner et Simonot (2001) et de Morlaix et Le Mener (2015) qui se réfèrent à la théorie de l'autodétermination de Deci et Ryan (1985), et d'autre part de ceux de Galand et Vanlede (2004) et de Lardy, Bressoux et Lima (2015) qui mobilisent le concept de sentiment d'efficacité personnelle développé par Bandura (1997). Enfin, le choix d'appuyer notre travail sur la «dynamique motivationnelle» de Viau (1998) se justifie par le fait que cet auteur mobilise lui-même cette théorie dans différents écrits portant sur l'enseignement supérieur (Bédard \& Viau, 2000; Viau \& Joly, 2001). sur le type d'approche de l'apprentissage pour lequel opte l'étudiant (Ramsden, 1988; Wolfs, 2007) et par ce biais, supposément, sur ses manières d'étudier. Néanmoins, à notre connaissance, aucune recherche empirique récente ne s'est attachée, dans le contexte francophone, à mesurer l'effet des pratiques pédagogiques des enseignants sur la motivation, ou encore les manières d'étudier des jeunes entrant à l'université.

En effet, l'implication des pratiques pédagogiques sur la scolarité des étudiants inscrits en première année universitaire reste pour l'heure trop peu documentée par la recherche francophone. On ne sait pas réellement, par exemple, quelle pratique au sens précis du terme engendre quels effets sur les variables préalablement citées. Il s'agit donc là d'un axe à développer (Romainville \& Michaut, 2012), certains considérant même que «le développement d'études en pédagogie universitaire pourrait contribuer à la réussite des étudiants» (Pageau \& Médaille, 2005).

Par ailleurs, l'impact des pratiques pédagogiques sur la scolarité des étudiants peut être abordé selon plusieurs angles. Il est d'une part possible de s'intéresser à l'effet des pratiques que l'on pourrait qualifier d'« observées » ou bien de «constatées » pour reprendre le terme de Clanet (2001), c'est-à-dire effectivement mises en œuvre in situ par les enseignants. Cela reviendrait à examiner quelles pratiques sont concrètement mobilisées par ces derniers en cours et à les mettre en rapport, par le biais d'analyses multi-niveaux, avec certaines variables liées aux étudiants, comme leurs notes aux examens. Mais il est intéressant, d'autre part, d'approcher le problème sous un autre angle, celui des pratiques telles qu'elles sont perçues par les étudiants. C'est sur cette seconde dimension, ayant déjà fait l'objet de recherches dans les pays anglo-saxons (Lizzio, Wilson \& Simons, 2002) mais très peu en France, que nous focalisons notre attention dans cet article. En effet, les étudiants arrivant à l'université découvrent des situations pédagogiques inédites, notamment en cours magistral (Boyer, Coridian \& Erlich, 2001), type de cours souvent mentionné comme facteur d'échec à I'université (Bruter, 2008). Les pratiques des enseignants durant les cours magistraux sont décrites par Clanet (2001), Boyer et Coridian (2002) ou bien encore plus récemment par Duguet (2014) comme s'apparentant majoritairement à des monologues sans interaction, ou en d'autres termes comme relevant des «méthodes traditionnelles » au travers desquelles l'enseignement est centré sur l'enseignant et non sur I'apprenant. Comme I'indiquent Duguet et Morlaix

.


(2012), on peut alors s'interroger quant aux effets sur la scolarité des étudiants (en termes de motivation, de manières d'étudier et de réussite) de la manière dont ils perçoivent les pratiques mobilisées par les enseignants lors de ce type de cours. Nous considérons que les pratiques pédagogiques des enseignants peuvent se référer à plusieurs dimensions, telles que la façon d'interagir avec les étudiants, le matériel utilisé pour enseigner, la façon d'organiser et de transmettre le contenu du cours, ou encore l'attitude de l'enseignant durant le cours. C'est ainsi plus précisément la perception des étudiants quant à ces différentes dimensions des pratiques qui est questionnée dans cette recherche. L'objectif ici n'est donc pas d'effectuer un état de l'art de la motivation des étudiants, ni de décrire leurs manières d'étudier, mais plutôt d'examiner comment la façon de percevoir les pratiques des enseignants peut engendrer des effets sur ces variables et par ce biais sur la réussite. Ce travail s'inscrit en ce sens davantage dans le prolongement des recherches portant sur les déterminants de la réussite universitaire que dans celui des recherches portant sur les modalités d'étude des jeunes inscrits en première année. Nous formulons pour hypothèse que plus un étudiant perçoit les pratiques de ses enseignants en cours magistral de façon positive, plus il fera preuve de motivation ${ }^{2}$, mobilisera des manières d'étudier propices à la réussite et verra ses chances de réussir en première année augmenter. Ce terme de «perception» sera préféré à ceux d'«opinion » ou d'«appréciation » qui feraient trop penser à un jugement de valeur.

\section{Démarche empirique}

Pour éclairer les liens supposés entre les variables mentionnées ci-dessus, nous avons mené une enquête par questionnaire sur le site de l'université de Bourgogne auprès d'étudiants de première année inscrits au sein des filières droit, administration économique et sociale

2 Le terme de motivation sera préféré à celui de mobilisation ou d'engagement universitaire. En effet, nous nous appuyons sur la théorie de l'autodétermination (Deci \& Ryan, 1985) et la théorie du sentiment d'efficacité personnelle (Bandura, 1997) qui induisent le fait que les besoins et perceptions des étudiants d'eux-mêmes, mais aussi du contexte dans lequel ils évoluent, ont un impact sur leur motivation. De ce fait, en référence aux écrits de Viau (1998) qui s'inscrivent dans le prolongement de ces théories, nous définissons la motivation comme étant « un état dynamique qui a ses origines dans les perceptions qu'un élève a de lui-même et de son environnement et qui l'incite à choisir une activité, à s'y engager et à per-
(AES), psychologie, sociologie et langues, littératures et civilisations étrangères (LLCE) anglais. Certes, ces filières tendent toutes à relever du domaine des sciences humaines, à tendance appliquée. Néanmoins, leur choix se justifie par le fait qu'elles accueillent un grand nombre d'étudiants présentant une hétérogénéité à la fois en termes d'origine sociale et de passé scolaire. De plus, elles proposent aux étudiants un nombre important de cours magistraux lors des deux semestres. Les passations directes, sous forme papier, se sont tenues durant des cours magistraux en novembre 2012. 734 étudiants, soit $36,7 \%$ de la population initialement visée par l'enquête ${ }^{3}$, ont répondu au questionnaire 4 .

En vue d'établir un lien entre la motivation, les manières d'étudier et les pratiques telles que perçues par les étudiants, et de pouvoir appréhender ces dimensions de manière plus synthétique, nous avons construit à partir des réponses des étudiants trois types de scores sur $100^{5}$ :

- un score de motivation : plus il est élevé, plus forte est la motivation de l'individu;

- un score de manières d'étudier : plus il est élevé, plus les individus ont opté pour des pratiques d'étude considérées comme a priori favorables à la réussite; - un score de "perception des pratiques» : construit à I'appui des variables de la dernière rubrique du questionnaire, plus celui-ci est proche de 100, plus il signifie que les pratiques des enseignants en cours magistral sont perçues positivement par les étudiants. C'est le poids qu'exerce ce score sur la motivation, les manières d'étudier et la réussite que nous souhaitons appréhender par le biais de nos analyses.

Ce que nous cherchons à mesurer n'est donc pas l'impact des pratiques pédagogiques en tant que telles, considérées indépendamment les unes des autres, mais bien leur effet au travers de la manière dont elles sont globalement perçues par les étudiants. Ce score de «perception des pratiques» représente en quelque sorte "l'avis moyen» des étudiants concernant l'ensemble des pratiques de leurs enseignants en cours magistral. Certes, on peut supposer que la perception des pratiques est susceptible de différer en

3 Il est intéressant de noter ici que ce taux signifie également qu'à peine plus d'un étudiant sur 3 était présent en cours lors des passations.

4 Nous invitons le lecteur à consulter le questionnaire joint en annexe 2 .

5 Pour plus d'informations sur la constitution de ces scores, nous invitons le lecteur à se référer à l'annexe 1 


\section{Le questionnaire}

Le questionnaire était articulé autour de cinq parties. Les deux premières concernaient les caractéristiques sociodémographiques des étudiants, leurs conditions de vie et leur passé scolaire. La troisième partie avait trait à la motivation des étudiants et comprenait, en référence aux travaux de Deci et Ryan (1985), Bandura (1997) et Viau (1998), des questions destinées à appréhender l'état de la motivation des étudiants. La partie suivante, créée à partir des travaux de Lahire (1997), Millet (2003), Alava (2000) ou encore Soulié (2002), était consacrée aux manières d'étudier des étudiants. Enfin, la dernière section portait sur leur perception des pratiques pédagogiques en cours magistral. Ils ont été invités à indiquer s'ils jugeaient les interactions avec les enseignants suffisantes et satisfaisantes, le matériel et les supports employés bien adaptés, l'organisation du cours cohérente, le rythme du cours adapté à leurs besoins, les enseignants suffisamment clairs dans leur façon de s'exprimer, la façon de transmettre le cours motivante, l'attitude de l'enseignant engageant à être plus attentif, mais aussi si de façon globale ils considéraient que les pratiques de leurs enseignants avaient un impact positif sur leur motivation et facilitaient leurs apprentissages.

Les étudiants ont été priés d'indiquer leur nom ou numéro d'étudiant sur le questionnaire, cela afin de pouvoir apparier les données les concernant avec leurs résultats aux examens.

fonction des unités d'enseignement et donc des enseignants qui assurent le cours. Mais pour des raisons à la fois déontologiques et matérielles, nous n'avons pu interroger les étudiants sur les pratiques de chacun de leurs enseignants. Nous avons donc fait le choix de raisonner sur la perception des pratiques des enseignants dans leur ensemble.

Enfin, les résultats des étudiants aux examens ont été collectés sur la base de données APOGEE 6 . La réussite est de ce fait appréhendée à travers la note moyenne obtenue aux examens du premier et du second semestre et la validation de l'année. Ce dernier indicateur est dichotomisé et sont pris en compte deux groupes d'étudiants : ceux autorisés à passer en deuxième année, composé des individus admis ou $\mathrm{AJAC}^{7}$, soit 486 étudiants $(66,2 \%)$, et ceux auxquels l'accès à la deuxième année de licence est refusé, regroupant les individus ajournés ou défaillants ${ }^{8}$, soit 218 étudiants (29,8\%).

\section{Les étudiants de l'échantillon : description et perception des pratiques pédagogiques}

Il aurait été intéressant de tenir compte dans ce travail de la filière d'inscription des étudiants, notamment

6 APOGEE : application pour l'organisation et la gestion des enseignements et des étudiants. Ce logiciel permet de gérer les inscriptions et dossiers de l'ensemble des étudiants inscrits à l'université.

7 Ajournés, autorisés à continuer.

8 Les termes «ajourné» et "défaillant» sont employés par les services administratifs de l'université. Un étudiant est déclaré «ajourné» lorsque ses résultats aux examens sont trop faibles pour lui permettre d'accéder à l'année supérieure et « défaillant» en cas d'absence aux examens. parce qu'il est maintenant reconnu que celle-ci constitue une matrice de socialisation aux codes du travail universitaire (Millet, 2003). Toutefois, les faibles effectifs interrogés au sein des filières LLCE et AES ne nous permettent pas de construire des modèles d'analyse statistiquement valides. Nous faisons donc le choix méthodologique de raisonner sur l'ensemble des individus de l'échantillon. Celui-ci est constitué aux trois quarts de femmes $(74,1 \%)$, d'individus de nationalité française $(96,7 \%)$ et célibataires $(90,2 \%)$. Les modes d'habitat dominants sont le logement en location $(35,1 \%)$ et chez les parents $(27,4 \%)$. Le chef de famille est le plus souvent cadre ou de profession intellectuelle supérieure ( $23 \%)$, de profession intermédiaire $(19,6 \%)$ ou ouvrier (18,4\%). Un peu plus de la moitié des étudiants sont boursiers et $84,6 \%$ n'ont aucune activité salariée en dehors des heures de cours. $72,8 \%$ n'ont pas redoublé durant leur scolarité primaire et secondaire. En revanche, un quart étaient déjà inscrits dans l'enseignement supérieur l'année précédente. Les étudiants issus de séries générales du baccalauréat constituent à eux seuls plus de $80 \%$ de l'échantillon. Moins de $15 \%$ ont obtenu la mention bien ou très bien au baccalauréat.

La motivation des étudiants de l'échantillon semble dans l'ensemble relativement forte : la moyenne des scores de motivation est relativement élevée puisqu'elle se situe à 75,64, pour un écart-type de 14,78 . Cela s'explique par le fait que, dans le cadre de notre enquête, n'ont été interrogés que les étudiants présents en cours environ deux mois après la rentrée. On peut imaginer que puisqu'ils continuent d'assister aux cours magistraux alors que ceux-ci n'ont aucun caractère obligatoire, ils font preuve d'une certaine motivation pour leurs études. En ce qui 
Tableau 1. Perception des pratiques pédagogiques par les étudiants

\begin{tabular}{|c|c|c|c|}
\hline Items & OUI & NON & Non renseigné \\
\hline Les interactions avec les enseignants sont suffisantes & $54,6 \%$ & $43,9 \%$ & $1,5 \%$ \\
\hline Les interactions avec les enseignants sont satisfaisantes & $65,9 \%$ & $32,4 \%$ & $1,6 \%$ \\
\hline Le matériel et les supports employés sont bien adaptés & $72,2 \%$ & $25,7 \%$ & $2 \%$ \\
\hline L'organisation des cours magistraux par les enseignants semble cohérente & $86,5 \%$ & $11,2 \%$ & $2,3 \%$ \\
\hline Le rythme du cours est adapté aux besoins des étudiants & $75,1 \%$ & $23,2 \%$ & $1,8 \%$ \\
\hline Les enseignants sont suffisamment clairs & $79,9 \%$ & $17,4 \%$ & $2,7 \%$ \\
\hline La façon de transmettre est motivante pour l'étudiant & $68,4 \%$ & $28,8 \%$ & $2,9 \%$ \\
\hline L'attitude des enseignants conduit à être plus attentif & $68,8 \%$ & $28,2 \%$ & $3 \%$ \\
\hline
\end{tabular}

concerne en revanche les scores de manières d'étudier, la moyenne se situe 56,42 pour un écart-type égal à 14,54 , indiquant ainsi que les étudiants n'optent pas massivement pour des manières d'étudier réellement favorables à leur réussite. Ces résultats semblent cohérents puisque les jeunes inscrits en première année, en comparaison à la scolarité vécue au lycée, se trouvent confrontés dès leur arrivée à l'université à un grand nombre de bouleversements, notamment du point de vue du travail personnel qu'ils ont à fournir. II leur incombe de décrypter les codes implicites du travail intellectuel exigé à l'université (Coulon, 2005). Cependant, cette tâche n'est pas sans poser problème à un grand nombre d'individus.

Les étudiants portent dans l'ensemble un regard plutôt positif sur les pratiques des enseignants en cours magistral (voir tableau 1) ${ }^{9}$. Plus de la moitié des étudiants, voire parfois plus de quatre individus sur cinq, ont répondu favorablement aux items proposés. $56,7 \%$ des étudiants ont considéré que les pratiques pédagogiques avaient globalement un impact positif sur leur motivation et $65,4 \%$ qu'elles facilitaient leurs apprentissages.

Comme indiqué dans la partie précédente, afin d'obtenir une vision plus synthétique de la perception des étudiants, nous avons construit un score de «perception des pratiques». Le score moyen concernant cette variable est relativement élevé : 68,14 pour un

9 Les étudiants n'ont pas été interrogés concernant les modalités de contrôle des connaissances. II nous a semblé que le contrôle terminal ne constituait pas une pratique pédagogique au sens où nous définissons ce terme. L'une des limites de cette recherche tient néanmoins au fait que nous n'avons pas pris en compte la pratique consistant à procéder à une évaluation formative des acquis des étudiants pour les soutenir dans la progression de leurs apprentissages. écart-type à 24,9, traduisant ainsi une perception dans l'ensemble plutôt positive des pratiques pédagogiques des enseignants. De tels résultats paraissent surprenants dans la mesure où d'autres travaux (Mucchielli, 1998; Erlich, 1998; Coulon \& Paivandi, 2008) ont montré que régnait parfois une incompréhension entre enseignants et étudiants. Cependant, ce manque de synergie relèverait davantage d'une dimension relationnelle que d'une dimension pédagogique, comme l'évoquent Mucchielli (1998) en montrant que plus de $40 \%$ des étudiants mentionnent le «contact avec les enseignants » comme étant source de difficultés à l'université, ou plus récemment Coulon et Paivandi (2008) selon qui l'étudiant entrant à l'université est amené à « réinterpréter ses rapports avec l'enseignant dans un cadre nouveau». D’ailleurs, selon Bru (2004), les étudiants ne portent finalement pas un jugement particulièrement négatif sur la manière dont «l'enseignement universitaire » est dispensé. Néanmoins, les résultats présentés ici sont à relativiser puisque seuls les étudiants présents lors des cours magistraux ont complété le questionnaire. Il aurait été intéressant de pouvoir questionner les étudiants absents afin de savoir si les pratiques pédagogiques des enseignants constituent l'un des motifs de leur non-assiduité. De même, on peut se demander si les étudiants sont réellement satisfaits des pratiques de leurs enseignants ou s'ils s'en déclarent satisfaits parce qu'ils n'ont en réalité pas véritablement le choix : on peut imaginer qu'ils entrent à l'université avec des a priori sur le déroulement d'un cours magistral. Ignorant le fait que d'autres types de pratiques existent, cette situation les conduirait à estimer que celles mises en œuvre par les enseignants sont conformes aux méthodes généralement mobilisées 


\begin{tabular}{|c|c|c|c|}
\hline \multirow[b]{2}{*}{ Modalité de référence } & \multirow[b]{2}{*}{ Modalité active } & \multicolumn{2}{|c|}{$\begin{array}{l}\text { Modèle } 1 \\
N=728\end{array}$} \\
\hline & & Coefficient & Significativité \\
\hline \multicolumn{4}{|c|}{ Caractéristiques sociodémographiques, conditions de vie } \\
\hline \multirow{2}{*}{$\begin{array}{l}\text { Catégorie socioprofessionnelle (CSP) } \\
\text { du chef de famille favorisée }\end{array}$} & CSP du chef de famille défavorisée & $-0,21$ & ns \\
\hline & CSP du chef de famille moyenne & $-0,72$ & $* *$ \\
\hline \multirow[t]{2}{*}{ Logement chez les parents } & Logement en résidence universitaire & 0,017 & ns \\
\hline & Autre type de logement & 0,073 & $* *$ \\
\hline \multicolumn{4}{|l|}{ Passé scolaire } \\
\hline \multirow[t]{3}{*}{ Bac $S$} & Bac littéraire & $-0,089$ & $* *$ \\
\hline & Bac ES & $-0,068$ & * \\
\hline & $\begin{array}{l}\text { Bac technologique, professionnel ou } \\
\text { autre }\end{array}$ & $-0,123$ & *** \\
\hline Pas étudiant l'an passé & Déjà étudiant l'an passé & $-0,054$ & * \\
\hline \multicolumn{2}{|l|}{ Score de manières d'étudier } & 0,464 & *** \\
\hline \multicolumn{2}{|l|}{ Score de perception des pratiques } & 0,247 & $* * *$ \\
\hline \multicolumn{2}{|l|}{ Constante } & \multicolumn{2}{|l|}{39541} \\
\hline \multicolumn{2}{|l|}{ R- ajusté } & \multicolumn{2}{|l|}{$32,5 \%$} \\
\hline
\end{tabular}

Lecture : le modèle de régression linéaire permet d'estimer l'effet net d'une variable en raisonnant «toutes choses égales par ailleurs». Le score de motivation constitue dans ce tableau la variable dépendante, c'est-à-dire à expliquer. À titre d'exemple, à CSP, logement, passé scolaire, manières d'étudier et perception des pratiques identiques, le fait d'être titulaire d'un baccalauréat technologique ou professionnel diminue de 0,123 point (valeur du coefficient de régression) le score de motivation en comparaison à un individu ayant obtenu un baccalauréat scientifique. De même, un point de plus au score de perception des pratiques apporte 0,247 point de plus au score de motivation. L'effet des coefficients est à examiner au regard de leur significativité qui se lit comme suit : ***: $1 \%$; ** $5 \%$; $: 10 \%$; ns > $10 \%$ (ou autrement dit non significatif). Le R- ajusté (coefficient de détermination) indique la part de variance expliquée par l'ensemble des variables intégrées dans le modèle : ici elles permettent d'expliquer 32,5\% de la variance du score de motivation. Le modèle a été réalisé à partir des données relatives à un effectif de 728 étudiants.

durant ce type de cours et donc satisfaisantes. Néanmoins, ces résultats conduisent également à s'interroger quant aux effets de cette perception des pratiques sur la scolarité des étudiants.

\section{Effet statistique de la «perception des pratiques " sur la motivation et les manières d'étudier}

Même si l'on peut s'interroger sur le double sens des relations entre les variables considérées ${ }^{10}$, nous faisons le choix de nous intéresser plus particulièrement dans

10 Cela conduirait à supposer qu'un étudiant motivé et faisant usage de manières d'étudier propices à la réussite aurait une perception davantage positive des pratiques enseignantes qu'un individu qui serait peu motivé et qui rencontrerait des difficultés à s'organiser dans son travail personnel. ce travail aux effets de la perception des pratiques enseignantes par les étudiants sur leur motivation et leurs manières d'étudier. De ce fait, nous avons construit différents modèles de régression linéaire. Au contraire des tests de corrélations qui permettent uniquement d'examiner l'existence d'une relation linéaire significative entre deux variables, ce type de modèle permet d'expliciter les relations causales entre les variables. En d'autres termes, il permet de donner du sens aux relations existant entre les variables. Le premier modèle (voir tableau 2) a pour but de mettre au jour l'effet, toutes choses égales par ailleurs, de la perception des pratiques enseignantes sur la motivation des étudiants (ces deux variables étant, rappelons-le, traduites par des scores) ${ }^{11}$. Il se traduit donc comme suit :

11 Nous avons au préalable testé l'effet de différentes variables de contrôle, liées aux caractéristiques sociodémographiques, aux conditions de vie et au passé scolaire des étudiants sur leur moti- 
Motivation $=\mathrm{f}$ (caractéristiques sociodémographiques, conditions de vie, manières d'étudier, perception de pratiques)

Les résultats indiquent que les caractéristiques sociodémographiques des étudiants et leurs conditions de vie jouent un rôle mineur sur leur motivation. Parmi les variables liées au passé scolaire, seules deux exercent un impact significatif. Ainsi, en comparaison des bacheliers scientifiques, les titulaires d'un baccalauréat littéraire, économique et social, ainsi que professionnel ou technologique voient leur motivation diminuer. C'est concernant ces derniers ${ }^{12}$ que cet effet est le plus important, un tel constat pouvant sans doute s'expliquer par le fait que la poursuite d'études à l'université ne constitue pas pour ces bacheliers la voie «naturelle» (Ryk, 2012). Les manières d'étudier constituent la variable la plus explicative de la motivation des étudiants puisqu'un point de plus au score de manières d'étudier augmente celui relatif à la motivation de 0,464 point. En d'autres termes, plus les étudiants optent pour des manières d'étudier favorables à leurs apprentissages, plus ils sont motivés. Pour expliquer une telle relation, on peut supposer que plus l'étudiant s'investit dans son travail personnel, plus il a un fort sentiment d'être compétent dans la réalisation de ce travail, sa motivation s'en trouvant alors accrue si l'on se réfère aux théories de la motivation de Deci et Ryan (1985), de Bandura (1997) et de Viau (1998). Par ailleurs, cet investissement dans son travail personnel peut être vu comme le reflet d'un sentiment selon lequel l'étudiant estime que ce travail est utile à accomplir et y accorde une certaine valeur, cette perception constituant d'après Viau (1998) I'un des principaux déterminants de la motivation.

Mais surtout, le modèle 1 indique que la perception des pratiques exerce un effet positif et très significatif sur la motivation des étudiants. Un point de plus au score de perception des pratiques apporte 0,247 point de plus au score de motivation : plus un étudiant perçoit les pratiques pédagogiques de façon positive, plus il est motivé.

L'impact, toutes choses égales par ailleurs, de la perception par les étudiants des pratiques pédagogiques sur leurs manières d'étudier est en revanche tout autre (voir tableau 3$)^{13}$.

vation. Seules celles exerçant un effet significatif sur la motivation ont ensuite été intégrées au modèle présenté dans cet article.

12 Pour des raisons d'ordre méthodologique, nous avons regroupé dans notre analyse les détenteurs d'un baccalauréat technologique et ceux d'un baccalauréat professionnel. Ce sont donc ces deux catégories réunies qui sont concernées par ce résultat.
Les hommes obtiennent, toutes choses égales par ailleurs, un score de manières d'étudier inférieur à celui des femmes (-0,178 point). En d'autres termes, les femmes optent davantage que les hommes pour des manières d'étudier favorables à leur réussite. Ce résultat s'explique sans doute par le fait que Frickey et Primon (2003) décrivent les filles comme étant plus studieuses et les garçons comme adoptant un comportement plus «minimaliste», moins favorable à la réussite. Ce modèle indique également que les bacheliers littéraires bénéficient d'un léger avantage ( $+0,096$ point) en comparaison des bacheliers scientifiques. Rappelons que les filières enquêtées dans le cadre de notre travail se réfèrent davantage à des disciplines littéraires que scientifiques. Il paraît alors cohérent de considérer que les étudiants titulaires d'un baccalauréat littéraire ont développé des méthodes de travail qui sont plus en adéquation avec ce qui est attendu d'eux en première année. Par ailleurs, les individus s'étant vus décerner la mention bien ou très bien au baccalauréat mobilisent plus souvent des manières d'étudier favorables à leur réussite, en comparaison de ceux ayant obtenu la mention passable. On peut alors supposer ici que l'attribution d'une «bonne» mention au baccalauréat est elle-même liée aux pratiques d'étude adoptées au lycée. Ces étudiants auraient déjà dans le secondaire appris à apprendre "efficacement». D'autres facteurs exercent un effet positif sur les manières d'étudier, comme le redoublement dans le primaire ou le secondaire. Ce résultat, pourtant contreintuitif, s'explique sans doute par le fait que l'année redoublée leur a possiblement permis d'acquérir une certaine maturité et des méthodes de travail correspondant davantage à ce qu'une scolarité à l'université implique en termes de travail personnel. De même, le fait d'avoir déjà été étudiant l'année précédente joue un effet positif sur les manières d'étudier. Ce constat est vraisemblablement lié à une plus grande familiarisation avec le système d'enseignement supérieur et en ce sens avec les codes implicites du travail universitaire. La motivation constitue quant à elle un facteur fortement explicatif des manières d'étudier : à caractéristiques identiques, un point de plus au score de motivation augmente de 0,492 point celui de manières d'étudier. Autrement dit, plus un étudiant est motivé,

préalablement testé l'effet de diverses variables de contrôle sur les manières d'étudier des étudiants. Puis nous avons inclus dans le modèle 2 uniquement celles exerçant un effet significatif sur ce facteur. 
Tableau 3. Effet statistique de la «perception des pratiques» sur les manières d'étudier des étudiants

\begin{tabular}{|c|c|c|c|}
\hline \multirow[b]{2}{*}{ Modalité de référence } & \multirow[b]{2}{*}{ Modalité active } & \multicolumn{2}{|c|}{$\begin{array}{l}\text { Modèle } 2 \\
N=728\end{array}$} \\
\hline & & Coefficient & Significativité \\
\hline \multicolumn{4}{|l|}{ Caractéristiques sociodémographiques } \\
\hline Femme & Homme & $-0,178$ & $* * *$ \\
\hline \multicolumn{4}{|l|}{ Passé scolaire } \\
\hline \multirow[t]{3}{*}{ Bac $S$} & Bac littéraire & 0,096 & $* *$ \\
\hline & Bac ES & 0,007 & ns \\
\hline & $\begin{array}{l}\text { Bac technologique, professionnel } \\
\text { ou autre }\end{array}$ & 0,027 & ns \\
\hline \multirow[t]{2}{*}{ Mention passable au Bac } & Mention assez bien & 0,007 & ns \\
\hline & Mention bien ou très bien & 0,117 & *** \\
\hline $\begin{array}{l}\text { Pas de redoublement dans le primaire } \\
\text { ou secondaire }\end{array}$ & $\begin{array}{l}\text { Au moins un redoublement dans } \\
\text { le primaire ou secondaire }\end{array}$ & 0,067 & $* *$ \\
\hline Pas étudiant l'an passé & Déjà étudiant l'an passé & 0,102 & *** \\
\hline \multicolumn{2}{|l|}{ Score de motivation } & 0,484 & $* * *$ \\
\hline \multicolumn{2}{|l|}{ Score de perception des pratiques } & $-0,024$ & ns \\
\hline \multicolumn{2}{|l|}{ Constante } & \multicolumn{2}{|l|}{20573} \\
\hline \multicolumn{2}{|l|}{ R- ajusté } & \multicolumn{2}{|l|}{$29,9 \%$} \\
\hline
\end{tabular}

Lecture : L'effet des coefficients est à examiner au regard de leur significativité qui se lit comme suit : ${ }^{* *}: 1 \%$; ${ }^{* *}: 5 \%$; $: 10 \%$; ns > $10 \%$ (ou autrement dit non significatif). Le R- ajusté (coefficient de détermination) indique la part de variance expliquée par l'ensemble des variables intégrées dans le modèle.

plus il opte pour des manières d'étudier favorables à sa réussite. Ce constat rejoint celui de Lambert-Le Mener (2012) selon qui le «profil motivationnel» des étudiants est lié à leurs pratiques d'étude, les plus motivés optant pour les pratiques d'étude réclamant le plus grand investissement de leur part. En ce qui concerne la perception des pratiques, à caractéristiques identiques, elle n'exerce aucun effet sur les manières d'étudier des étudiants : le coefficient associé à cette variable $(-0,024)$ est non significatif.

Toutefois, nous avons également construit un modèle de régression destiné à expliquer, toutes choses égales par ailleurs, le score des manières d'étudier, mais excluant la motivation comme variable explicative $^{14}$. Dans ce cas, la perception des pratiques est associée à un coefficient positif $(+0,127)$ et significatif au seuil de $1 \%$. Cela signifie qu'en l'absence de la motivation comme variable de contrôle, la perception des

14 Le modèle construit est donc le suivant : Score de manières d'étudier $=f$ (caractéristiques sociodémographiques, conditions de vie, passé scolaire, score de perception des pratiques). pratiques enseignantes par les étudiants joue un rôle positif sur leurs manières d'étudier : plus cette perception est positive, plus les manières d'étudier privilégiées par les étudiants sont propices à leur réussite. En conséquence, notre hypothèse mérite d'être nuancée : si aucun effet direct n'est démontré, on peut en revanche postuler l'existence d'un effet indirect de la perception des pratiques enseignantes sur les manières d'étudier transitant par la motivation des étudiants.

\section{Effet de la «perception des pratiques » sur la réussite}

L'effet de la perception des pratiques pédagogiques par les étudiants a également été testé, toutes choses égales par ailleurs, sur leur réussite, c'est-à-dire de façon plus précise sur la note moyenne obtenue au semestre 1 (modèle 3) et au semestre 2 (modèle 4) (voir tableau 4).

La perception des pratiques joue, toutes choses égales par ailleurs, un faible rôle sur la réussite au premier semestre, le coefficient associé à ce facteur étant 


\begin{tabular}{|c|c|c|c|c|c|}
\hline \multirow{3}{*}{ Modalité de référence } & \multirow[b]{3}{*}{ Modalité active } & \multirow{2}{*}{\multicolumn{2}{|c|}{$\begin{array}{c}\text { Modèle } 3 \\
\text { Semestre } 1(N=688)\end{array}$}} & & \\
\hline & & & & \multicolumn{2}{|c|}{$\begin{array}{c}\text { Modèle } 4 \\
\text { Semestre } 2(N=629)\end{array}$} \\
\hline & & Coefficient & Significativité & Coefficient & Significativité \\
\hline \multicolumn{6}{|c|}{ Caractéristiques sociodémographiques, conditions de vie } \\
\hline Femme & Homme & $-0,080$ & *** & $-0,108$ & ns \\
\hline \multirow[t]{2}{*}{$\begin{array}{l}\text { Logement chez } \\
\text { les parents }\end{array}$} & $\begin{array}{l}\text { Logement en résidence } \\
\text { universitaire }\end{array}$ & $-0,020$ & ns & $-0,020$ & ns \\
\hline & Autre type de logement & 0,009 & ns & 0,060 & ns \\
\hline \multicolumn{6}{|l|}{ Passé scolaire } \\
\hline \multirow[t]{3}{*}{ Bac $S$} & Bac littéraire & $-0,277$ & *** & $-0,284$ & *** \\
\hline & Bac ES & $-0,236$ & *** & $-0,184$ & ** \\
\hline & $\begin{array}{l}\text { Bac technologique, } \\
\text { professionnel ou autre }\end{array}$ & $-0,370$ & *** & $-0,329$ & $* * *$ \\
\hline $\begin{array}{l}\text { Bac obtenu } \\
\text { au rattrapage }\end{array}$ & $\begin{array}{l}\text { Bac obtenu en } 1^{\text {re }} \\
\text { session }\end{array}$ & 0,132 & $* * *$ & 0,105 & $* * *$ \\
\hline \multirow{2}{*}{$\begin{array}{l}\text { Mention passable au } \\
\text { Bac }\end{array}$} & Mention assez bien & 0,147 & $* * *$ & 0,139 & ** \\
\hline & $\begin{array}{l}\text { Mention bien } \\
\text { ou très bien }\end{array}$ & 0,355 & *** & 0,324 & *** \\
\hline $\begin{array}{l}\text { Pas de redoublement } \\
\text { dans le primaire ou } \\
\text { secondaire }\end{array}$ & $\begin{array}{l}\text { Au moins un } \\
\text { redoublement dans le } \\
\text { primaire ou secondaire }\end{array}$ & $-0,151$ & $* * *$ & $-0,139$ & $* * *$ \\
\hline Pas étudiant l'an passé & Déjà étudiant l'an passé & 0,149 & $* * *$ & 0,150 & $* * *$ \\
\hline \multicolumn{2}{|l|}{ Score de motivation } & 0,132 & $* * *$ & 0,105 & $* * *$ \\
\hline \multicolumn{2}{|c|}{ Score de manières d'étudier } & 0,089 & ** & 0,060 & ns \\
\hline \multicolumn{2}{|c|}{ Score de perception des pratiques } & 0,072 & ** & 0,134 & * \\
\hline \multicolumn{2}{|l|}{ Constante } & \multicolumn{2}{|l|}{6815} & \multicolumn{2}{|l|}{7063} \\
\hline \multicolumn{2}{|l|}{ R- ajusté } & \multicolumn{2}{|l|}{$41,1 \%$} & \multicolumn{2}{|l|}{$36,6 \%$} \\
\hline
\end{tabular}

Lecture : L'effet des coefficients est à examiner au regard de leur significativité qui se lit comme suit : ***: $1 \%$; **: $5 \%$; $: 10 \%$; ns > $10 \%$ (ou autrement dit non significatif). Le R- ajusté (coefficient de détermination) indique la part de variance expliquée par l'ensemble des variables intégrées dans le modèle.

peu élevé $(0,072)$. Cet effet perd d'ailleurs en significativité au second semestre. $A$ contrario, les variables liées au passé scolaire de l'étudiant demeurent des facteurs dont l'effet sur les notes aux examens est important. II en est de même pour le score de motivation.

La construction d'un modèle de régression logistique binaire ${ }^{15}$, ayant pour variable à expliquer le fait de valider ou non l'année, conforte ces résultats : la

15 Le modèle de régression logistique binaire fonctionne selon le même type de principe que le modèle de régression linéaire, mais est employé lorsque la variable dépendante est dichotomique, soit ici «année validée» ou «année non validée». Chaque modalité des variables explicatives se voit associer un coefficient qui s'inter- façon dont les étudiants perçoivent les pratiques de leurs enseignants ne constitue pas un facteur réellement explicatif de la probabilité qu'ils ont de valider I'année. Autrement dit, le fait d'avoir une perception davantage positive des pratiques pédagogiques ne conférerait pas réellement de chances en plus de valider l'année. On peut toutefois se demander si l'effet de ce facteur ne serait pas, là encore, absorbé par d'autres variables. Par conséquent, afin d'explorer davantage les relations indirectes entretenues entre ces différents facteurs, nous avons construit un modèle d'analyse en pistes causales (voir encadré).

Dans la figure 1, les liens significatifs entre les variables sont matérialisés par des flèches en caractère 


\section{L'analyse en pistes causales}

Autrement appelé modèle de cheminement ou encore path analysis, ce type de modèle présente l'intérêt de pouvoir estimer l'effet direct d'une variable indépendante $(X)$ (ici la perception des pratiques), sur une variable dépendante (Y) (la note au semestre 1). Mais il permet également de connaître l'effet indirect exercé par la variable indépendante sur la variable dépendante, transitant par une variable médiatrice (M) (la motivation et les manières d'étudier). À travers ces analyses, on distingue des variables de causes et des variables d'effets, chacune des flèches partant de la cause pour aller vers l'effet (Meuret \& Morlaix, 2006). À titre d'exemple, on considérera que la flèche partant de la variable «perception des pratiques» et allant à la «motivation » traduit l'effet de cette perception sur la motivation (voir figure 1).

Figure 1. Analyse en pistes causales mettant en lien perception des pratiques, note moyenne au semestre 1 , motivation et manières d'étudier

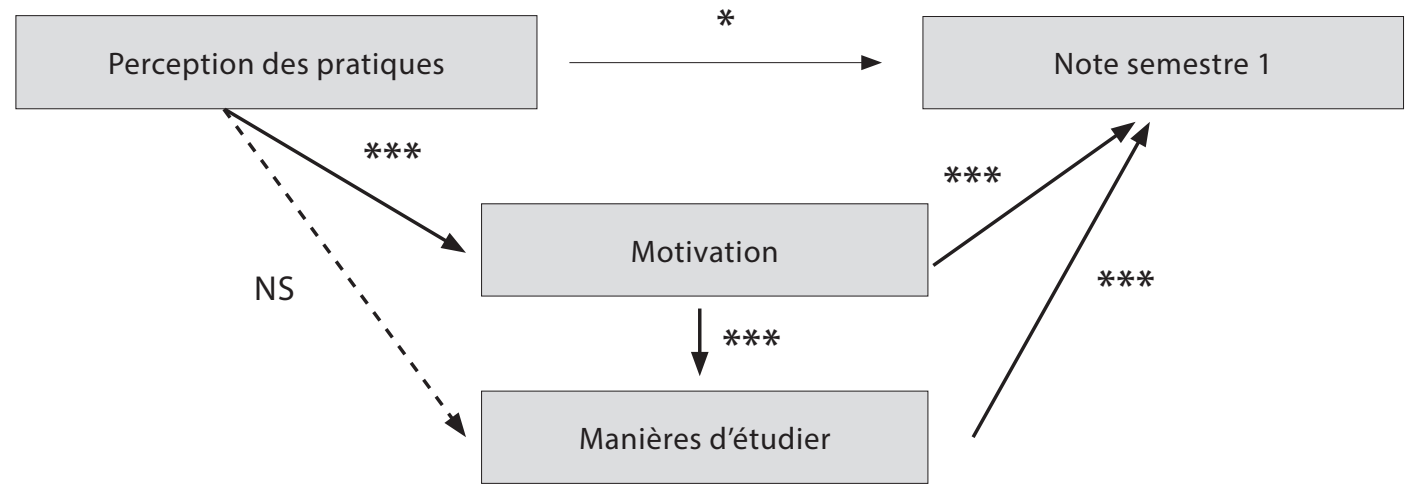

gras, tandis que l'absence de relation significative est représentée par une flèche en pointillés. Ce modèle confirme l'hypothèse d'un effet indirect de la perception des pratiques sur les manières d'étudier transitant par la motivation, ces deux dernières variables étant extrêmement liées. Autrement dit, la perception des pratiques joue sur la motivation des étudiants et par cet intermédiaire sur leurs manières d'étudier. II permet également de valider le postulat selon lequel l'impact de la perception des pratiques sur la note moyenne du premier semestre transite par la motivation des étudiants : plus les étudiants ont une perception favorable des pratiques de leurs enseignants, plus ils sont motivés et meilleure est la note moyenne obtenue au premier semestre. Ce type de résultat est identique au second semestre.

La figure 1 permet d'effectuer un autre constat intéressant : il y a un lien direct, mais peu significatif, entre la perception des pratiques et la note du premier semestre, alors que les modèles de régression linéaire permettaient de mettre en avant un effet, toutes choses égales par ailleurs, significatif au seuil de $5 \%$. Cette différence s'explique sans doute par la prise en compte de variables de contrôle dans les modèles de régression linéaire.

Enfin, le même type d'analyse a été effectué concernant la note moyenne obtenue au second semestre : la perception des pratiques joue dans ce cas un rôle très significatif (seuil de $1 \%$ ). Comme en témoignent les modèles de régression linéaire présentés précédemment, cet effet tend toutefois à s'atténuer, voire à disparaître lorsqu'est adopté un raisonnement «toutes choses égales par ailleurs».

\section{Conclusion}

La démocratisation et la massification de l'accès à l'université au cours du siècle dernier ont eu entre autres conséquences une hausse croissante des taux d'échec des étudiants inscrits en première année. Bien qu'une littérature abondante ait été produite sur le thème des déterminants de la réussite, rares sont les travaux à avoir procédé, dans le contexte français, à une mesure de l'effet des pratiques enseignantes et plus précisément de la manière dont elles sont perçues par les étudiants sur la scolarité de ces derniers. Pourtant, la 
question des pratiques pédagogiques à l'université demeure cruciale, le comité de suivi de la licence et de la licence professionnelle préconisant une "évolution » des pratiques enseignantes et une valorisation de la fonction pédagogique puisqu'il s'agit là d'éléments « indispensables à la réussite et à la qualité des formations de l'enseignement supérieur» (Raby, 2011). Or, nos analyses montrent que la perception des pratiques pédagogiques par les étudiants a un effet significatif, toutes choses égales par ailleurs, sur la motivation et indirectement, par le biais de cette variable, sur les manières d'étudier et la réussite. Néanmoins, il serait intéressant d'examiner ces résultats au regard de la filière d'inscription, variable non intégrée à nos modèles d'analyse pour des raisons statistiques.

Cette recherche s'inscrivant dans une démarche empirique novatrice dans le contexte français se heurte donc à certaines limites. Pour des raisons matérielles et organisationnelles, les données sur lesquelles reposent nos analyses ont été recueillies sur un seul site universitaire, au sein de cinq filières uniquement. Élargir le terrain d'enquête permettrait davantage de représentativité des données. D'ailleurs, l'article de Weisser, Gangloff-Ziegler et Hermann (2014) montre que la perception de la qualité d'une formation diffère entre les étudiants d'IUT et ceux de licence. Il serait alors intéressant d'examiner si la perception des pratiques enseignantes des individus inscrits en filière sélective varie fortement de celle d'étudiants des filières «classiques» de l'université et de mesurer, dans une perspective comparative, quel peut en être le lien avec leurs scolarités respectives. De plus, investiguer auprès $d^{\prime}$ 'individus inscrits au sein d'autres composantes que celles relatives aux sciences humaines permettrait sans doute une compréhension plus fine, à la fois descriptive et analytique, du rôle de la perception des pratiques enseignantes sur la scolarité des jeunes entrant à l'université.

En outre, les indicateurs et scores mobilisés sont critiquables. Le choix de la prise en compte de la moyenne obtenue au premier et au second semestre comme indicateur de réussite, plutôt que la note moyenne aux différentes unités d'enseignement ou aux différentes disciplines, est discutable. La mesure de la motivation employée dans ce travail demande de surcroît à être approfondie et perfectionnée. Il en est de même des scores traduisant les manières d'étudier et la perception des pratiques : leur construction est critiquable et demande à être améliorée. D'ailleurs, traduire de tels concepts au travers de scores peut s'avérer réducteur.
Néanmoins, les résultats mis en évidence ici présentent l'intérêt d'apporter un éclairage nouveau sur certains mécanismes à l'œuvre dans la compréhension de la réussite en première année universitaire. Ils posent par ailleurs question en plusieurs sens : les effets de la perception des étudiants à l'égard des pratiques des enseignants en cours magistral sur leur scolarité sont-ils semblables en ce qui concerne les travaux dirigés et les travaux pratiques? En effet, les modalités pédagogiques étant davantage semblables à celles connues au lycée, on peut faire l'hypothèse que les pratiques mobilisées durant ces types de cours sont encore plus favorablement perçues par les étudiants. De plus, nos résultats indiquent que les étudiants ont dans l'ensemble une opinion favorable des pratiques des enseignants. Mais concrètement, quelles sont ces dernières? Alors que l'avenir du cours magistral est régulièrement remis en question (en témoigne un récent numéro de la revue Distance et médiation des savoirs intitulé "Le cours magistral a-t-il un avenir?», paru en 2015), quelles sont les pratiques mobilisées aujourd'hui par les enseignants durant ce type de cours? C'est d'ailleurs à cette question que nous avons tenté de répondre, au travers d'une recherche récente portant sur la description des pratiques des enseignants universitaires (Duguet, 2014). Nous avons ainsi pu montrer que malgré une certaine hétérogénéité des pratiques, celles-ci tendent majoritairement à relever des méthodes traditionnelles. Mais dans ce cas, comment expliquer qu'un tel type de pratiques soit perçu de façon positive par les étudiants? D'autres recherches empiriques pourraient permettre d'alimenter davantage le débat sur la question.

De même, comme évoqué plus haut, les pratiques des enseignants peuvent être abordées sous un autre angle, celui des pratiques "observées», autrement dit effectivement mobilisées par les enseignants durant les cours. Quelles sont leurs implications sur la scolarité des étudiants? Les effets des pratiques pédagogiques constituant aujourd'hui encore une "zone d'ombre» (Michaut, 2012), une telle question demeure d'actualité, à I'heure où nombre de recherches remettent en cause les pratiques d'enseignement mobilisées durant les cours magistraux (Raby, 2011 ; Paivandi, 2012) et où la formation à la pédagogie des enseignants, reposant sur la base du volontariat, fait toujours débat.

Amélie Duguet Université de Bourgogne, IREDU (EA 7318) amelie.duguet@u-bourgogne.fr 
ALAVA S. (2000). «Les profils d'auto-direction et les pratiques d'études des étudiants en première année d'université». Les Sciences de l'éducation - Pour l'Ėre nouvelle, vol. 33, $\mathrm{n}^{\circ} 1$, p. 43-71.

BANDURA A. (1997). Self-efficacy: the exercise of control. New York : Freeman.

BEAUD S. (2008). «Enseignement supérieur, la "démocratisation scolaire" en panne ». Formation emploi, n¹01, p. 149-165.

BEAUD S. \& PIALOUX M. (2001). «Les"bacs pro" à I'université. Récit d'une impasse». Revue française de pédagogie, $\mathrm{n}^{\circ}{ }^{136}$, p. 87-95.

BEAUPÈRE N., CHALUMEAU L., GURY N. \& HUGRÉE C. (2007). L'abandon des études supérieures. Paris : La Documentation française.

BÉDARD D. \& VIAU R. (2001). Le profil d'apprentissage des étudiantes et des étudiants de l'université de Sherbrooke. Résultats de l'enquête menée au trimestre d'automne 2000. Manuscrit non publié. Sherbrooke (Canada) :Vice-rectorat à l'enseignement.

BÉDUWÉ C. \& GIRET J. F. (2004). «Le travail en cours d'études a-t-il une valeur professionnelle?».Économie et statistique, vol.378, n¹, p.55-83.

BERTRAND C. (2014). Soutenir la transformation pédagogique dans l'enseignement supérieur. Paris : Ministère de l'Enseignement supérieur et de la Recherche.

BOULET A., SAVOIE-ZAJC L. \& CHEVRIER J. (1996). Les stratégies d'apprentissage à l'université. Sainte-Foy : Presses de I'Université du Québec.

BOYER R. \& CORIDIAN C. (2002). «Transmission des savoirs disciplinaires dans l'enseignement universitaire, une comparaison histoire-sociologie». Sociétés contemporaines, $n^{\circ} 48$, p. 41-61.

BOYER R., CORIDIAN C. \& ERLICH V. (2001). «L'entrée dans la vie étudiante. Socialisation et apprentissages». Revue française de pédagogie, $\mathrm{n}^{\circ} 136$, p. 97-105.

BRU M. (2004). «Pratiques enseignantes à l'université : opportunité et intérêt des recherches». In E. Annoot \& M.-F. Fave-Bonnet (dir.), Pratiques pédagogiques dans l'enseignement supérieur : enseigner, apprendre, évaluer. Paris : L'Harmattan, p.17-36.

BRUTER A. (2008). «Le cours magistral comme objet d'histoire». Histoire de l'éducation, $n^{\circ} 120$, p. 2-19.

CLANET J. (2001). «Étude des organisateurs des pratiques enseignantes à l'université ». Revue des sciences de l'éducation, vol.27, n², p.327-352.

CONVERT B. (2010). «Espace de l'enseignement supérieur et stratégies étudiantes». Actes de la recherche en sciences sociales, $n^{\circ} 183$, p. 14-31.

COULON A. (2005). Le métier d'étudiant : l'entrée dans la vie universitaire. Paris : Economica, $2^{\mathrm{e}}$ éd.

COULON A. \& PAIVANDI S. (2008). État des savoirs sur les relations entre les étudiants, les enseignants et les IATOSS dans les établissements d'enseignement supérieur. Paris : Observatoire national de la vie étudiante.

DANNER M. (1999). Améliorer la réussite en premier cycle universitaire : évaluation externe des effets du tutorat d'accompagnement. Thèse de doctorat, sciences de l'éducation, université de Bourgogne.

DE KETELE J.-M. (2010). «La pédagogie universitaire, un courant en plein développement ». Revue française de pédagogie, $n^{\circ} 172$, p.5-13.

DECI E. L. \& RYAN R. M. (1985). Intrinsic motivation and selfdetermination in human behavior. New York: Plenum press.

DELHAXHE M., HOUART M. \& POLLET M.-C. (2011). «Comment développer des compétences méthodologiques et organisationnelles?». In P. Parmentier (dir.), Recherches et actions en faveur de la réussite en première année universitaire. Namur : Presses universitaires de Namur, p.61-70.

DUGUET A. (2014). Les pratiques pédagogiques en première année universitaire: description et analyse de leurs implications sur la scolarité des étudiants. Thèse de doctorat, sciences de l'éducation, université de Bourgogne.

DUGUET A. \& MORLAIX S. (2012). «Les pratiques pédagogiques des enseignants à l'université : quelle variété pour quelle efficacité?» Questions vives, vol. 6, n 18.

DURU-BELLAT M. (1995). «Des tentatives de prédiction aux écueils de la prévention en matière d'échec en première année d'université». Savoir, n³, p.399-416.

ERLICH V. (1998). Les nouveaux étudiants: un groupe social en mutation. Paris : Armand Colin.

FELOUZIS G. (2001). «Les délocalisations universitaires et la démocratisation de l'enseignement supérieur». Revue française de pédagogie, $\mathrm{n}^{\circ} 136$, p.53-65.

FONTAINE S. \& PETERS M. (2012). «L'abandon des étudiants à l'université : état de la question». In M. Romainville \& C. Michaut (dir.), Réussite, échec et abandon dans l'enseignement supérieur. Bruxelles : De Boeck, p.33-52.

FORNER Y. \& SIMONOT C. (2001). «Motivation et adaptation à I'université». Psychologie et psychométrie, vol.22, $\mathrm{n}^{\circ} 1$, p. 59-73.

FRICKEY A. \& PRIMON J.-L. (2003). «Manières d'étudier en première année d'université et passage dans l'année supérieure». In G. Felouzis (dir.), Les mutations actuelles de l'université. Paris : PUF, p. 229-249.

GALAND B., NEUVILLE S. \& FRENAY M. (2005). «L'échec à l'université en Communauté française de Belgique : comprendre pour mieux prévenir?». Cahiers de recherche en éducation et formation, $\mathrm{n}^{\circ} 39$, p. 5-17.

GALAND B. \& VANLEDE M. (2004). «Le sentiment d'efficacité personnelle dans l'apprentissage et la formation : quel rôle joue-t-il? D'où vient-il? Comment intervenir?». Savoirs, $\mathrm{n}^{\circ} 5$ (hors série), p. 91-116.

GRUEL L. (2002). «Les conditions de réussite dans l'enseignement supérieur». OVE infos, n², p. 1-10. 
GURY N. (2007). «Les sortants sans diplôme de l'enseignement supérieur : temporalités de l'abandon et profils des décrocheurs». L'Orientation scolaire et professionnelle, vol.36, $n^{\circ} 2$, p. 2-16.

HARTER S. (1981). «A new self-report scale of intrinsic versus extrinsic orientation in the classroom: Motivational and informational components ». Developmental Psychology, $\mathrm{n}^{\circ} 17, \mathrm{p} .300-312$.

LAHIRE B. (1997). Les manières d'étudier. Paris : La Documentation française.

LAMBERT-LE MENER M. (2012). La performance académique des étudiants en première année universitaire: influence des capacités cognitives et de la motivation. Thèse de doctorat, sciences de l'éducation, université de Bourgogne.

LARDY L, BRESSOUX P. \& LIMA L. (2015). «Les facteurs qui influencent la réussite des étudiants dans une filière universitaire technologique : le cas de la première année d'études en DUT GEA ». L'Orientation scolaire et professionnelle, vol. 44, $\mathrm{n}^{\circ} 4$, p.543-578.

LIZZIO A., WILSON K. \& SIMONS R. (2002). «University students' perceptions of the learning environment and academic outcomes ». Studies in Higher Education, vol.27, $\mathrm{n}^{\circ} 1$, p. 27-52.

M'PIAYI A. (1999). Les déterminants de la réussite, le cas du Deug B à l'Université de Bourgogne. Thèse de doctorat, sciences de l'éducation, université de Bourgogne.

MÉNARD L. (2012). «Apprentissage en classe et persévérance au premier cycle universitaire». In M. Romainville \& C. Michaut (dir.), Réussite, échec et abandon dans l'enseignement supérieur. Bruxelles : De Boeck, p.177-198.

MEURET D. \& MORLAIX S. (2006). «L'influence de l'origine sociale sur les performances scolaires : par où passe-telle?» Revue française de sociologie, vol. 47, $\mathrm{n}^{\circ} 1$, p. 49-79.

MICHAUT C. (2000). L'influence du contexte universitaire sur la réussite des étudiants. Thèse de doctorat, sciences de l'éducation, université de Bourgogne.

MICHAUT C. (2012). «Réussite, échec et abandon des études dans l'enseignement supérieur français : quarante ans de recherche». In M. Romainville \& C. Michaut (dir.), Réussite, échec et abandon dans l'enseignement supérieur. Bruxelles: De Boeck, p.53-68.

MILLET M. (2003). Les étudiants et le travail universitaire :étude sociologique. Lyon : Presses universitaires de Lyon.

MILLET M. (2012). "L'"échec" des étudiants de premiers cycles dans l'enseignement supérieur en France. Retours sur une notion ambiguë et descriptions empiriques». In M. Romainville \& C. Michaut (dir.), Réussite, échec et abandon dans l'enseignement supérieur. Bruxelles : De Boeck, p.69-88.

MINISTÈRE DE L'ENSEIGNEMENT SUPÉRIEUR ET DE LA RECHERCHE [MESR] (2013). L'état de l'enseignement supérieur et de la recherche en France, $\mathrm{n}^{\circ} 6$.

MORLAIX S. \& LE MENER M. (2015). «La motivation des étudiants à l'entrée à l'université : quels effets directs ou indirects sur la réussite?». Recherches en éducation, $\mathrm{n}^{\circ} 22$,
MORLAIX S. \& SUCHAUT B. (2012). "Les déterminants sociaux scolaires et cognitifs de la réussite en première année universitaire». Revue française de pédagogie, $\mathrm{n}^{\circ} 180$, p.77-94.

MUCCHIELLI L. (1998). «La pédagogie universitaire en question : le point de vue des étudiants de premier cycle en psychologie ». Recherche et formation, n²9, p.161-176.

NICOURD S., SAMUEL O. \& VILTER S. (2011). «Les inégalités territoriales à l'université : effets sur les parcours des étudiants d'origine populaire». Revue française de pédagogie, $\mathrm{n}^{\circ} 176$, p. 27-40.

PAGEAU D. \& MÉDAILLE C. (2005). «La recherche institutionnelle au Québec ». In P. Chenard \& P. Doray (dir.), L'enjeu de la réussite dans l'enseignement supérieur. Sainte-Foy : Presses de I'Université du Québec, p.111-126.

PAIVANDI S. (2012). «Pour réussir la transition entre secondaire et supérieur ». Cahiers pédagogiques, $n^{\circ} 25$, hors série numérique, p. 22-25.

PIROT L. \& DE KETELE J.-M. (2000). «L'engagement académique de l'étudiant comme facteur de réussite à l'université, étude exploratoire menée dans deux facultés contrastées ». Revue des sciences de l'éducation, vol. $26, \mathrm{n}^{\circ} 2$, p.367-394.

RABY G. (2011). Pour une licence attractive doublement qualifiante, permettant une poursuite d'études en Master et une insertion professionnelle, une licence assurant un parcours de réussite aux étudiants. Rapport d'enquête. Paris : Comité de Suivi de la Licence et de la Licence professionnelle.

RAMSDEN P. (1988). Improving learning: New perspective. Londres : Nichols Pub Co.

ROMAINVILLE M. (2000). L'échec dans l'université de masse. Paris : L'Harmattan.

ROMAINVILLE M. \& MICHAUT C. (dir). (2012). Réussite, échec et abandon dans l'enseignement supérieur. Bruxelles : De Boeck.

RYK G. (2012). «"Décrocheurs" en L1 à l'université Paul Verlaine-Metz». Relief, n³6, p.47-60.

SÉNÉCAL C., VALLERAND R. J. \& PELLETIER L. G. (1992). «Les effets du type de programme universitaire et du sexe de l'étudiant sur la motivation académique». Revue des sciences de l'éducation, $n^{\circ} 18$, p. 375-388.

SOLOMON D. \& KENDALL A. J. (1976). «Individual characteristics and children's performances in "open" and "traditional" classroom settings ». Journal of Educational Psychology, $n^{\circ} 68$, p. 613-625.

SOULIÉ C. (2002). "L'adaptation aux "nouveaux publics" de l'enseignement supérieur : auto-analyse d'une pratique d'enseignement magistral en sociologie». Sociétés contemporaines, vol.4, n³8, p.11-39.

VALLERAND R. J. (1993). «La motivation intrinsèque et extrinsèque en contexte naturel : implication pour les secteurs de l'éducation, du travail, des relations interpersonnelles et des loisirs». In R. J. Vallerand \& E. E. Thill (dir.), Introduction à la psychologie de la motivation. Laval : Études vivantes, p.533-581. 
VIAU R. (1998). La motivation en contexte scolaire. Bruxelles : De Boeck, $2^{\mathrm{e}}$ éd.

VIAU R. \& JOLY J. (2001) «Comprendre la motivation à réussir des étudiants universitaires pour mieux agir». Communication au colloque de I'ACFAS. En ligne : <http:// www.uquebec.ca/ uss1109/dossiers/Acfas_Viau.pdf> (consulté le 14 mars 2013).
WEISSER M., GANGLOFF-ZIEGLER C. \& HERMANN H. (2014). «Perception de la qualité d'une formation universitaire par les étudiants : étude comparative selon le mode d'orientation et l'ancienneté dans le cursus». Mesure et évaluation en éducation, vol.37, nº1, p. 83-108.

WOLFS J.-L. (2007). Méthodes de travail et stratégies d'apprentissage : du secondaire à l'université : Recherche, théorie, application. Bruxelles : De Boeck, $3^{\mathrm{e}}$ éd.

\section{Annexes}

\section{Annexe 1. La construction des scores de motivation, de manières d'étudier et de perception des pratiques pédagogiques}

Pour construire ces scores, nous avons d'abord attribué pour toutes les questions entre 0 et 3 points selon la réponse cochée par l'étudiant. À titre d'exemple, pour la question « Vous sentez-vous globalement bien intégré à l'université», destinée à servir d'indicateur de la motivation, nous avons attribué 0 point lorsque l'étudiant répondait par «non pas du tout» (traduisant une absence de motivation), 1 point pour «plutôt non», 2 points pour « plutôt oui » et 3 points pour « oui tout à fait» (traduisant une forte motivation). Pour les questions à réponse dichotomique, nous avons attribué 0 ou 3 points en fonction de la réponse cochée : par exemple, pour l'item concernant le rattrapage des cours auxquels l'étudiant a été absent, nous avons attribué 0 point lorsque l'étudiant répondait «non» et 3 points lorsqu'il répondait «oui ». Enfin, pour les questions à trois modalités de réponse, nous avons accordé $0,1,5$ ou 3 points selon la modalité de réponse, comme pour le sentiment de compétence pour réussir : nous avons accordé 0 point à une réponse négative de la part de l'étudiant, 1,5 point pour la modalité «ne sait pas» et 3 points à toute réponse positive.

Le choix des modalités de réponse auxquelles ont été attribués des points s'est effectué à la lumière de la littérature mentionnée dans l'article. Ainsi, si l'on prend pour exemple l'utilité des enseignements, Viau (1998) montre que la perception de la valeur d'une activité joue un rôle déterminant sur la motivation. De ce fait, nous avons accordé 3 points lorsque l'individu accordait une certaine valeur aux enseignements et aucun point lorsqu'il considérait ces derniers sans utilité. Cette mise en regard avec les écrits scientifiques a été effectuée pour chaque question.

Nous avons appliqué cette même méthodologie pour la construction du score de motivation et celle du score de manières d'étudier.

En référence à certains travaux développant le concept de motivation (Deci \& Ryan, 1985; Bandura, 1997; Viau, 1998), les variables mobilisées pour le calcul du score de motivation, renvoyant aux questions du questionnaire, sont les suivantes:

- sentiment d'avoir été assez renseigné concernant les possibilités de poursuite d'études post baccalauréat;

- préférence pour un autre type d'orientation;

- hésitation avant d'effectuer le choix de la filière;

- sentiment de compétence pour réussir dans la filière;

- utilité des enseignements (pour obtenir la licence, dans l'exercice du futur métier);

- connaissance des modalités d'examen;

- sentiment d'intégration à l'université;

- fréquence des absences en cours magistral;

- rattrapage des cours auxquels l'étudiant n'a pas assisté;

- nombre d'heures hebdomadaires de travail personnel;

- période de révision (pendant ou à la fin du semestre);

- habitudes de travail (se fixer des objectifs pour réviser, respecter ces objectifs, persévérer face aux difficultés)

- comportement durant les cours magistraux. 
De même, nous avons réalisé une recension des travaux portant sur les manières d'étudier favorables à la réussite $^{16}$, s'appuyant notamment sur les écrits de Boulet, Savoie-Zajc et Chevrier (1996), Lahire (1997), Pirot et De Ketele (2000), Boyer et Coridian (2002), Soulié (2002) et Frickey et Primon (2003), nous conduisant à mobiliser les variables suivantes pour le calcul du score de manières d'étudier :

- mise en place d'une tactique/stratégie pour réussir;

- absence de tout appareil audio/vidéo lors des révisions;

- organisation du travail personnel (fréquence de fréquentation de la BU/BS, fréquence à laquelle l'étudiant reste travailler à l'université en dehors des heures de cours, fréquence du travail pendant les week-ends, fréquence du travail pendant les vacances);

- consultation et tenue d'un agenda;

- façon de travailler les cours (recopiage des cours au propre, action de faire ou refaire des exercices, action de faire des fiches de résumé);

- relecture des notes de la fois précédente avant d'aller en cours;

- lecture des textes assignés avant d'aller en cours;

- nombre de lectures effectuées depuis le début de l'année;

- habitudes lors de la lecture (action de souligner/surligner/encadrer, action de prendre des notes, identification des idées principales du texte);

- difficultés pour prendre des notes en cours magistral;

- façon de prendre des notes en cours magistral;

- méthode privilégiée pour réviser.

Enfin, pour la construction du score de perception des pratiques pédagogiques, nous avons appliqué le même raisonnement que précédemment ( 0 ou 3 points pour les réponses dichotomiques, 0, 1,5 ou 3 points pour les questions à 3 modalités de réponse et $0,1,2$ ou 3 points pour celles à 4 modalités). Nous avons pour cela mobilisé toutes les questions de la dernière partie du questionnaire, à l'exception des questions 55 et 66 concernant lesquelles il a été considéré que les réponses ne pouvaient être interprétées comme étant un avis plutôt positif ou non envers les pratiques des enseignants. Certes, les questions auxquelles ont eu à répondre les étudiants portaient davantage sur des dimensions des pratiques que sur des pratiques en tant que telles au sens précis du terme. Elles permettent tout de même d'obtenir un indice d'ordre général concernant le degré de satisfaction des étudiants vis-à-vis des pratiques des enseignants.

Pour chaque individu, nous avons additionné les points obtenus relativement aux variables liées à la motivation. Nous avons fait de même pour celles liées aux manières d'étudier et aux perceptions des pratiques pédagogiques, aboutissant ainsi à l'obtention de trois scores distincts. Ceux-ci ont ensuite été standardisés sur 100.

Aucune variable n'a été pondérée dans la constitution de ces scores. Ce choix se justifie par le fait qu'une pondération de certains items aurait requis au préalable de formuler des hypothèses selon lesquelles certaines variables permettent plus que d'autres de traduire la motivation et les pratiques d'étude des individus. Nous avons pour notre part décidé d'accorder la même importance à toutes les variables mobilisées dans la constitution de ces scores. 
Votre NOM ou votre NUMERO D'ÉTUDIANT

\section{VOUS ET VOTRE FAMILLE}

1- Votre sexe : $\square$ Masculin $\square$ Féminin

2- Votre année de naissance :

3- Votre nationalité :

4- Votre situation familiale :

$\begin{array}{lll}\square \text { Célibataire } & \square \text { En concubinage } & \square \text { Marié(e) } \\ \square \text { Pacsé(e) } & \square \text { Séparé(e) } & \square \text { Divorcé(e) }\end{array}$

5- Quelle est la profession de :

Votre père

Votre mère

6- Diplôme le plus élevé de vos parents (cochez la case correspondante) :

$\begin{array}{lcc}\text { Aucun } & \text { Père } & \text { Mère } \\ \text { Certificat d'études / BEPC / Brevet } & \square & \square \\ \text { CAP / BEP } & \square & \square \\ \text { Baccalauréat général } & \square & \square \\ \text { Baccalauréat technologique ou professionnel } & \square & \square \\ \text { Bac }+2 & \square & \square \\ \text { Bac }+3 \text { ou }+4 & \square & \square \\ \text { Bac }+5 \text { et }+ & \square & \square \\ \text { Ne sait pas } & \square & \square\end{array}$

Ne sait pas

7- Durant cette année universitaire, vous logez :

$\square$ En résidence universitaire du CROUS

$\square$ Logement en location

$\square$ En chambre chez l'habitant

$\square$ Chez vos parents

$\square$ En colocation

$\square$ Chez un autre membre de votre famille

$\square$ Autre :

8- Etes-vous boursier ?

$\square$ Oui $\square$ Non

9- Avez-vous un emploi en dehors des cours pendant l'année scolaire ?

$\square$ Oui $\square$ Non

10-Si oui, combien d'heures vous prend-il par semaine?

$\square 35 \mathrm{~h}$ et plus $\square 25$ à $34 \mathrm{~h} \quad \square 15$ à $24 \mathrm{~h} \quad \square 5$ à $14 \mathrm{~h} \quad \square$ Moins de $5 \mathrm{~h}$

11- Quelle est votre principale source de revenus mensuelle ?

$\begin{array}{ll}\square \text { Parents } & \square \text { Bourse } \quad \square \text { Autres, précisez } \\ \square \text { Travail d'été } & \square \text { Travail durant les études }\end{array}$

VOTRE PARCOURS SCOLAIRE

12- En quelle année avez-vous obtenu votre Bac?

13- Quel type de Bac avez-vous obtenu?

Baccalauréat général :

$\square \mathrm{L}$

$\square \mathrm{ES}$

$\square \mathrm{S}$

Baccalauréat technologique :

Baccalauréat professionnel :

$\square$

DAEU (équivalent au Bac): 
14- Avez-vous obtenu votre Bac à la première session ?

$\square$ Oui

$\square$ Non, je suis allé(e) au rattrapage

15- Quelle mention avez-vous obtenu au Bac?

$\square$ Aucune (mention passable)

$\square$ Mention assez bien

$\square$ Mention bien

$\square$ Mention très bien

16- Avez-vous redoublé durant votre scolarité à l'école primaire, au collège et au lycée ?

$\square$ Oui $\quad \square$ Non $\quad$ Si oui, à quel(s) niveau(x)?

17- Étiez-vous déjà étudiant l'an passé ?

$\square$ Oui $\quad \square$ Non $\quad$ Si oui, dans quelle école / filière ?

\section{VOTRE CHOIX DE L'UNIVERSITÉ / DE VOTRE FILIÈRE, VOTRE MOTIVATION}

18- Dans vos démarches concernant la poursuite d'études après le Bac, comment vous êtes-vous renseigné ? (plusieurs réponses possibles)

$\square$ Portes ouvertes des établissements

$\square$ Consulté un conseiller d'orientation

$\square \mathrm{CIO}$

$\square$ Recherches sur internet

$\square$ Salons de l'étudiant

$\square$ Demandé conseil aux amis, à la famille

$\square$ Brochures au CDI du lycée

$\square$ Cherché aucun renseignement

$\square$ Demandé conseil aux professeurs de lycée

19- Estimez-vous avoir été suffisamment renseigné ?

$\square$ Oui

$\square$ Non

20- Numérotez de 1 à 3 les trois principales raisons vous ayant conduit à vous inscrire à l'université :

\begin{tabular}{|l|l|}
\hline & Elle est proche de votre domicile \\
\hline & Vos amis y vont aussi \\
\hline & Votre famille vous a encouragé à y aller \\
\hline $\begin{array}{l}\text { Vous avez un projet professionnel précis qui ne peut se concrétiser } \\
\text { qu'en allant à l'université }\end{array}$ \\
\hline & Pour le plaisir et la satisfaction d'apprendre de nouvelles choses \\
\hline & Par défaut car vous n'avez pas été accepté dans une filière sélective \\
\hline $\begin{array}{l}\text { Par défaut car vous ne saviez pas trop où vous inscrire (n'aviez pas de } \\
\text { véritable projet) }\end{array}$ \\
\hline
\end{tabular}

21- Vous êtes allé à l'université, auriez vous préféré bénéficier d'un autre type d'orientation ?

$\square$ Oui

$\square$ Non

22- Si oui, vous auriez préféré aller en : (une seule réponse possible)

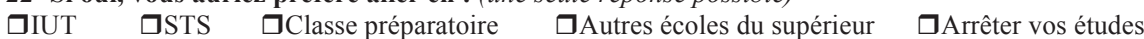

23- Avez-vous hésité avant d'effectuer le choix de cette filière ?

$\square$ Oui $\square$ Non

24- Pour quelle(s) raison(s) avez-vous choisi cette filière précisément ? Numérotez de 1 à 3 les trois principales raisons vous ayant conduit à vous inscrire dans cette filière.

\begin{tabular}{|l|l|}
\hline & Intérêt pour la discipline enseignée \\
\hline & Projet professionnel précis \\
\hline & Par curiosité \\
\hline & $\begin{array}{l}\text { Vous pensez qu'elle vous permettra d'obtenir un meilleur emploi / une } \\
\text { meilleure rémunération }\end{array}$ \\
\hline
\end{tabular}




\begin{tabular}{|l|l|}
\hline & Parce que vous réussissez dans cette discipline \\
\hline & Par défaut car vous ne saviez pas dans quelle voie poursuivre \\
\hline & $\begin{array}{l}\text { Par défaut, car vous n'avez pas pu intégrer une filière sélective, et cette } \\
\text { filière est celle qui s'en rapproche le plus }\end{array}$ \\
\hline & Cette filière vous a été conseillée par vos professeurs du lycée \\
\hline & Vos amis sont également inscrits dans cette filière \\
\hline & Votre famille a insisté pour que vous vous y inscriviez \\
\hline
\end{tabular}

25- Avez-vous le sentiment d'être suffisamment compétent pour réussir dans cette filière ?

$\square$ Oui $\quad \square$ Non $\square$ Ne sait pas

26- De façon générale, pensez-vous que les enseignements dispensés dans cette filière vous seront utiles :

- Pour obtenir votre licence $\square$ Oui $\square$ Non

- Dans l'exercice de votre futur métier $\quad \square$ Oui $\quad \square$ Non

27- Connaissez-vous la façon dont vos connaissances seront évaluées aux examens dans les différentes disciplines / matières ?

$\square$ Oui $\square$ Oui mais pas toutes $\square$ Non, je ne les connais pas

28- Vous sentez-vous globalement bien intégré à l'université ?

$\square$ Oui tout à fait $\square$ Plutôt oui $\square$ Plutôt non $\quad \square$ Non pas du tout

29- Actuellement, concernant votre motivation pour vos études, vous diriez que vous êtes :

$\square$ Pas motivé $\quad \square$ Peu motivé $\quad \square$ Assez motivé $\quad \square$ Très motivé

30- Par rapport au lycée, vous sentez-vous parfois « déstabilisé(e) » par les aspects suivants du fonctionnement universitaire? (Cochez les modalités qui ont pu vous déstabiliser, plusieurs réponses possibles)

$\square$ Les cours en amphithéâtre

$\square$ Votre emploi du temps (rythme, organisation)

$\square$ Les relations avec les enseignants

$\square$ La qualité de l'information (affichage, réunions de rentrée...)

$\square$ Le rapport à l'administration (inscription, renseignements...)

$\square$ L'anonymat (nombre d'étudiants, côté impersonnel de la fac...)

$\square$ La taille des locaux

$\square$ Aucun de ces aspects ne m'a déstabilisé

31- Depuis le début de l'année, avez-vous été absent(e) en cours magistral ?

口Jamais $\square$ Exceptionnellement $\square$ De temps en temps $\quad \square$ Souvent

32- Vous arrive-t-il d'être absent aux cours magistraux pour les raisons suivantes (Cochez, pour chaque proposition, la case correspondante) :

Votre emploi empiète sur vos heures de cours

Un cours ne vous intéresse pas

$\square$ Oui $\square$ Non

Vous n'appréciez pas la façon d'enseigner de l'enseignant $\quad \square$ Oui $\quad \square$ Non

Vous avez mieux à faire que de venir en cours $\quad \square$ Oui $\quad \square$ Non

Vous vous étiez couché tard la veille $\quad \square$ Oui $\square$ Non

33- Rattrapez-vous les cours auxquels vous n'avez pas assisté ?

$\square$ Oui $\quad \square$ Non

34- Participez-vous aux séances de tutorat?

$\square$ Oui $\square$ Non 
35- À l'heure actuelle, quel niveau d'études souhaitez-vous atteindre?

$\square \mathrm{Bac}+2$

$\square$ Master $(\mathrm{Bac}+5)$

$\square$ Licence $(\mathrm{Bac}+3)$

$\square$ Au-delà de $\mathrm{Bac}+5$

$\square$ Ne sait pas

\section{VOS MANIÈRES D'ÉTUDIER}

36- Combien d'heures par semaine (du lundi au dimanche) consacrez-vous en moyenne à votre travail personnel (pour étudier)?

$\square$ Aucune $\square$ Moins de 5 heures $\quad \square$ Entre 5 et 10 heures $\quad \square$ Plus de 10 heures

37- De façon générale, diriez-vous que vous avez mis au point une tactique de travail / stratégie afin de mieux réussir?

$\square$ Oui $\square$ Non

Si oui, laquelle?

38- De façon générale, vous diriez de vous que :

$\square$ Vous révisez vos cours durant tout le semestre

$\square$ Vous réviserez uniquement à l'approche des examens

39- Le plus souvent, dans quelle ambiance effectuez-vous votre travail personnel ? (Une seule réponse possible)

$\square$ Seul chez vous $\quad \square$ Seul, à la bibliothèque

$\square$ Chez vos amis, en leur compagnie

$\square$ Avec vos collègues à la bibliothèque

$\square$ Seul ou avec vos collègues dans un bar / cafétéria

40- Avez-vous pour habitude d'éteindre la télévision, la radio ou tout autre appareil audio/vidéo quand vous étudiez?

$\square$ Oui

$\square$ Non

41- Précisez la façon dont vous vous organisez pour votre travail personnel (pour étudier) (Cochez, pour chaque proposition, la case correspondante).

\begin{tabular}{|l|l|l|l|}
\cline { 2 - 4 } \multicolumn{1}{c|}{} & Souvent & $\begin{array}{c}\text { De temps en } \\
\text { temps }\end{array}$ & Jamais \\
\hline Vous travaillez à la BU* / BS** & & & \\
\hline $\begin{array}{l}\text { Il vous arrive de rester travailler à 1'Université } \\
\text { en dehors des heures de cours }\end{array}$ & & & \\
\hline Vous travaillez le week-end & & & \\
\hline Vous travaillez pendant les vacances scolaires & & & \\
\hline
\end{tabular}

* et ** : Bibliothèque universitaire et bibliothèque de section

42- Quand vous étudiez, avez-vous pour habitude de :

Consulter et tenir à jour un agend

Vous fixer des objectifs

$\square$ Oui $\square$ Non

De respecter ces objectifs

$\square$ Oui $\square$ Non

Persévérer lorsque vous rencontrez une difficulté $\quad \square$ Oui $\square$ Non

43- Sur quoi porte principalement votre travail personnel ? (Une seule réponse possible)

$\square$ Vos notes de cours

$\square$ Des livres, des articles

$\square$ Les polycopiés distribués par les enseignants 
44- Pour travailler vos cours, vous arrive-t-il (Cochez, pour chaque proposition, la case correspondante) :

\begin{tabular}{|l|l|l|l|}
\cline { 2 - 4 } \multicolumn{1}{c|}{} & \multicolumn{1}{c|}{ Souvent } & De temps en temps & Jamais \\
\hline De les relire seulement & & & \\
\hline De les recopier au propre & & & \\
\hline De faire (ou refaire) des exercices & & & \\
\hline De faire des fiches (pour les résumer) & & & \\
\hline
\end{tabular}

45- Avant d'assister aux cours, relisez-vous vos notes de la fois précédente?

$\square$ Souvent $\square$ De temps en temps $\square$ Jamais

46- Lisez-vous les textes qui vous ont été assignés avant d'aller en cours ?

$\square$ Souvent $\square$ De temps en temps $\square$ Jamais

47- Depuis le début de l'année scolaire, avez-vous lu (livres, articles... etc) pour vos études ?

$\square$ Non $\quad \square$ Oui entre 1 et 5 références $\quad \square$ Oui plus de 5 références

48- Lorsque vous lisez pour vos études, vous arrive-t-il de (Cochez, pour chaque proposition, la case correspondante) :

\begin{tabular}{|l|c|c|c|}
\cline { 2 - 3 } \multicolumn{1}{c|}{} & Souvent & $\begin{array}{c}\text { De temps en } \\
\text { temps }\end{array}$ & Jamais \\
\hline Souligner / surligner / encadrer certains passages & & & \\
\hline Prendre des notes & & & \\
\hline $\begin{array}{l}\text { Essayer d'identifier les idées principales et } \\
\text { dégager le contenu essentiel du texte }\end{array}$ & & & \\
\hline
\end{tabular}

49- Diriez-vous que vous fournissez plus d'efforts dans les matières / UE où vous pensez avoir plus de chances de réussir?

$\square$ Oui $\square$ Non

50- En cours magistral, avez-vous déjà rencontré des difficultés pour prendre des notes ?
$\square$ Oui, souvent
$\square$ Oui, parfois
$\square$ Non, pas trop
$\square$ Non, jamais

51- Concernant la prise de notes en cours magistral, vous : (une seule réponse possible)

$\square$ Notez tout ce que dit l'enseignant et ce qu'il affiche au tableau

$\square$ Ne copiez que les éléments qui vous paraissent essentiels

$\square$ Vous ne prenez aucune note (ou très peu)

52- Généralement, pendant le déroulement des cours magistraux :

$\square$ Vous n'écoutez pas vraiment l'enseignant (vous pensez à autre chose, parlez avec votre voisin, faites une autre activité... etc)

$\square$ Vous vous montrez attentif et essayez de comprendre le plus de choses possible

53- Généralement, quelle méthode privilégiez-vous (ou comptez-vous employer) pour réviser ? (une seule réponse possible)

$\square$ Vous vous basez sur vos notes de cours uniquement et essayez de les mémoriser par cœur. Tant pis si vous n'avez pas tout compris, l'essentiel est que vous réussissiez à retenir le contenu pour le citer le jour de l'examen.

$\square$ Vous travaillez sur vos notes de cours et effectuez des lectures complémentaires, reliez les idées nouvelles à ce que vous connaissez déjà. Votre attention est avant tout centrée sur la compréhension.

\section{LES PRATIQUES PÉDAGOGIQUES DE VOS ENSEIGNANTS}

Entendons par «pratique pédagogique » la façon dont l'enseignant interagit avec vous, le matériel qu'il utilise en cours (PowerPoint, polycopiés ...etc), la manière dont il organise le cours, sa façon de le transmettre... etc. 
54- Pensez-vous que les pratiques pédagogiques de l'ensemble de vos enseignants en cours magistral ont globalement un impact positif sur votre motivation?

$\square$ Oui

$\square$ Non

55- Pensez-vous que les pratiques pédagogiques de vos enseignants en cours magistral contribuent à modifier vos manières d'étudier? $\square$ Oui

$\square$ Non

56- Si oui, pensez-vous que de façon générale les pratiques pédagogiques de vos enseignants vous permettent d'acquérir des manières d'étudier favorisant votre réussite aux examens? $\square$ Oui $\square$ Non

57- Les interactions avec vos enseignants en cours magistral vous semblent-elles en règle générale :

- Suffisantes?

$\square$ Oui tout à fait

- Satisfaisantes?

$\square$ Oui tout à fait

$\square$ Plutôt oui

$\square$ Plutôt non

$\square$ Non pas du tout

Oui tout à fait

$\square$ Plutôt oui

$\square$ Plutôt non

$\square$ Non pas du tout

58- Diriez-vous que, de façon générale, le matériel et les supports employés par vos enseignants en cours magistral (tableau, PowerPoint, polycopiés ...etc) sont bien adaptés et facilitent vos apprentissages?

$\square$ Oui tout à fait

$\square$ Plutôt oui

$\square$ Plutôt non

$\square$ Non pas du tout

59- L'organisation globale des cours magistraux par les enseignants (gestion du temps imparti à la séance, présentation des objectifs en début de cours, récapitulatif de ce qui a été vu la fois précédente, pause entre 2 heures de cours ...etc) vous semble-t-elle généralement cohérente?

$\square$ Oui tout à fait $\square$ Plutôt oui $\square$ Plutôt non $\quad \square$ Non pas du tout

60- Les enseignants vous semblent-ils en règle générale adapter leur cours à vos besoins ?

$\square$ Oui tout à fait $\quad \square$ Plutôt oui $\quad \square$ Plutôt non $\quad \square$ Non pas du tout

61- De façon générale, le rythme des cours magistraux vous semble-t-il :

$\square$ Trop lent $\quad \square$ Correct $\square$ Trop rapide

62- Pensez vous que les enseignants sont globalement suffisamment clairs lors des cours magistraux (répètent plusieurs fois ce qui est difficile, reformulent les choses, mettent l'accent sur les points centraux ...etc) ?

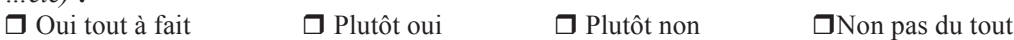

63- Pensez-vous qu'en cours magistral, en règle générale, la façon dont les enseignants transmettent leur cours (dictée du cours, utilisation de l'humour, utilisation d'exemples...) est motivante pour vous?

$\square$ Oui tout à fait $\quad \square$ Plutôt oui $\quad \square$ Plutôt non $\quad \square$ Non pas du tout

64- Considérez-vous que l'attitude des enseignants en cours magistral (intonations dans la voix, utilisation du langage corporel, déplacements dans l'amphithéâtre, maintien de l'ordre et de la discipline...etc) vous conduit à être généralement plus attentif en cours?
$\square$ Oui tout à fait
$\square$ Plutôt oui
$\square$ Plutôt non
$\square$ Non pas du tout

65- Diriez-vous que, de façon générale, les pratiques pédagogiques employées par vos enseignants en cours magistral facilitent vos apprentissages?
$\square$ Oui tout à fait
$\square$ Plutôt oui
$\square$ Plutôt non
$\square$ Non pas du tout

66- Pensez-vous qu'en fin de compte les pratiques pédagogiques employées par l'ensemble de vos enseignants en cours magistral soient susceptibles d'avoir un impact sur votre réussite aux examens ? $\square$ Oui $\square$ Non 